\title{
Vision-Aided Indoor Pedestrian Dead Reckoning
}

\author{
Jingjing YAN \\ International Doctoral \\ Innovation Centre \\ University of Nottingham \\ Ningbo 315100, China \\ Jingjing.YAN@nottingham. \\ edu.cn
}

\author{
Gengen HE \\ Department of Geographical \\ Science \\ University of Nottingham \\ Ningbo 315100, China \\ Gengen.He@nottingham.edu \\ .cn
}

\author{
Anahid BASIRI \\ Centre for Advanced \\ Analysis \\ University College London \\ London, UK \\ a.basiri@ucl.ac.uk
}

\author{
Craig Hancock \\ Department of Civil \\ Engineering \\ University of Nottingham \\ Ningbo 315100, China \\ Craig.Hancock@nottingham \\ .edu.cn
}

\begin{abstract}
Vision-aided inertial navigation has become a more popular method for indoor positioning recently. This popularity is basically due to the development of light-weighted and low-cost Micro Electro-Mechanical Systems (MEMS) as well as advancement and availability of CCD cameras in public indoor area. While the use of inertial sensors and cameras are limited to the challenge of drift accumulation and object detection in line of sight, respectively, the integration of these two sensors can compensate their drawbacks and provide more accurate positioning solutions. This study builds up upon earlier research on "Vision-Aided Indoor Pedestrian Tracking System", to address challenges of indoor positioning by providing more accurate and seamless solutions. The study improves the overall design and implementation of inertial sensor fusion for indoor applications. In this regard, genuine indoor maps and geographical information, i.e. digitized floor plans, are used for visual tracking application the pilot study. Both of inertial positioning and visual tracking components can work stand-alone with additional location information from the maps. In addition, while the visual tracking component can help to calibrate pedestrian dead reckoning and provides better accuracy, inertial sensing module can alternatively be used for positioning and tracking when the user cannot be detected by the camera until being detected in video again. The mean accuracy of this positioning system is $10.98 \%$ higher than uncalibrated inertial positioning during experiment.
\end{abstract}

Keywords-pedestrian dead reckoning; pedestrian detection; deep learning; sensor fusion; indoor optical positioning

\section{INTRODUCTION}

Recently, unavailability of Global Navigation Satellite Systems (GNSS) signals inside the building, where most of people's time are being spent, has resulted in the development and deployment of many indoor positioning technologies [1-4]. Only some of the most popular ones include Wi-Fi, Bluetooth Low Energy (BLE), Radio Frequency Identification Services (RFID), Ultra-Wide Band (UWB), Dead Reckoning (DR), and Optical Positioning systems. Although there is still no ideal solution to achieve similar level of robustness, accuracy, availability, continuity and reliability compared to, for example, Global Positioning System (GPS) as the most widely used outdoor positioning system $[5,6]$, the latter two technologies, i.e. Optical and DR positioning systems, are very promising techniques for future market for three main reasons;

International Doctoral Innovation Centre, Ningbo Education Bureau, Ningbo Science and Technology Bureau, and the University of Nottingham. (a) The advancement in the manufacturing of MEMS-based Inertial Measurement Units (IMU) and CCD cameras has led to a, cheaper, lower energy consuming, smaller and more precise products [7-12]. (b) The operation of these two positioning systems is flexible enough to be adjusted with respect to wide spectrum of applications and environments. This can be regarded as versatile methods for indoor positioning. (c) Both trends in the miniaturization of IMU sensors, which has made them commercialized for mass market smartphones, and also the ubiquity of surveillance cameras in public building area, have given a higher possibility of their use in every-day applications [13], with minimum of additional installation and modification of infrastructure or devices.

The Pedestrian Dead Reckoning (PDR) systems, which usually contain triad accelerometers and gyroscopes, can provide relative positioning information, orientation and velocity in Inertial Navigation Systems (INSs) for indoors [1418]. PDR-based methods can be divided into several categories depended on the location of IMU: foot-mounted [26, 27], handheld [28, 11], backpack [29-31] or head-mounted [32, 33, 24]. The accuracy of PDR solution could be compromised due to bias drift, i.e. the temporal accumulation of errors. This can challenge the use of long-term use of PDR on stand-alone basis and so some external position information are needed for calibration and providing the absolute location $[16,19,3,10$, 20]. These issues can be attenuated by system dynamics configuration $[21,22]$ or complementary sensing system [3, 20].

Meanwhile, the improving service quality of optical tracking system (OTS) has promoted many applications used by pedestrians, including indoor navigation [23]. The main advantage of introducing optical positioning is to feed enrich information, such as video data, to enable the object detection based on feature extraction. This has leaded to a much more accurate localization results [7, 23]. However, the performance of OTS will be easily compromised due to the ambient occlusion as OTS requires the line of sight (LOS) between camera and targets [24]. Conventional methods for motion estimation from camera are based on optical flow or feature detection [20]. The former, although with higher accuracy, could be more computationally expensive and may require the precise conditions of lighting and precise cameras. In addition, it also assumes that motions are small and limited enough to be 
ignored between frames [34], which might not be true in some real-world applications and scenarios. Feature-based methods are more common approach. They use landmarks, and thanks to the availability of indoor landmarks, they can provide solution in many indoor applications with relatively less computation power required $[35,20]$.

In order to counterbalance the drawbacks of these two positioning systems, this paper suggests the integration of them. The OTS, with a level of higher accuracy, can help to calibrate the accumulative error and bias of INS. While the INS can be address the challenge of LOS for OTS-based positioning, for the relatively short time intervals when the users cannot be viewed by cameras. The hybrid system is expected to enjoy the advantages of both two positioning systems, providing with higher overall accuracy, continuity, availability and reliability $[3,18]$. This kind of vision-aided INS has been commonly applied in many applications and areas of research, including robotics like Simultaneous Localization and Mapping (SLAM) and unmanned vehicle system, as it can provide with the 3D position and the orientation estimation in motion tracking [20, 25]. Many of similar implemented systems, use an attached monocular/stereo camera with IMU on a fixed platform, and integrate the IMU and camera data based on egomotion pose estimation, using variants of Kalman Filter (KF), such as Extended KF (EKF) and Unscented KF (UKF) [e.g. 8, 13, 35, 18, 24], Particle Filter (PF) [e.g. 36, 13] or slowing the sample rate of IMU data [37]. However, this paper proposes a prototype of hybrid system, which utilizes current infrastructures of surveillance system in public places and IMU sensors on user devices to provide user location, with the support of digitized floor plan in real coordinate system.

The proposed system utilizes a relatively different set-up, in comparison with currently used methods, to attach IMU and camera on the same platform. This paper proposes and implements a distributed platform. This physically and conceptually separates the positioning systems, i.e. different platforms for video data from surveillance camera data and IMU data from smartphones. In addition, it does not use the conventional landmark-based feature detection components for camera orientation estimation. The video data in this study are used for position estimation by deep-learning techniques based pedestrian detection with the combination of floor plan and camera location in built environment. The inertial data will also be pre-processed by transferring detected trajectory based on map information. Then the calculated 2D paths from video and INS are subsequently matched against their time steps for inertial positioning calibration purpose. Having inertial positing solution calibrated, both components can now work cooperatively and complementarily to provide a more accurate, continuous positioning service.

\section{SYSTEM DESIGN}

The proposed hybrid system has got two main positioning components; visual tracking and handheld PDR (Fig.1). The handheld PDR component works continuously, unlike the visual tracking component which only functions where LOS is available. The functionality of the system from the user perspective is such so that an Android phone records the accelerations and orientations during movements in order to get relative positions with poses. Once the camera detects the users, their positions are estimated by depth information in the frame. In order to calculate the absolute position, the digitized floor plans with georeferenced coordinates are fed into both of these components. This could be potentially very helpful for further development of the system and provide a seamless indooroutdoor positioning service. The visually tracking data are then used for calibration of the INS position solution. This prototype can help to improve the accuracy of PDR positioning. The calibrated PDR data are utilized to link the consecutive frames and make the trajectory of the user smoother and continuing between cameras frames or where the users are not detected in the video. The solution for user detection and the proposed scheme for tracking is explained in the next section.

\section{A. Pedestrian Detection Based Visual Tracking}

\section{1) Deep-Learning Based Pedestrian Detection}

The conventional methods of pedestrian tracking, which are generally feature based, can be divided into two modes - mobile and static. The former is used to localize the camera and the latter to localize the moving target in frames [38, 23]. Both modes require depth information to support transformation from image distance to actual distance in the scene. This transformation is solvable using a baseline from synthetic stereovision or from additional sensors like laser or range cameras [23]. However, feature based methods are limited to the extent of the applications and environments as their parameters need to be modified regularly based on ambient and environment to extract certain features. In this regard, deep learning methods can help with the ubiquity of the solution and improve the flexibility of use and surrounding environment. This study applies a method called Faster R-CNN (Fig.2), which is based on Regional Proposal Network (RPN) and Region-Based Convolutional Neural Networks (R-CNNs), providing with real-time and more accurate pedestrian detection possibility. The RPN is used for predicting Bounding Box (BB) and classifying objectness, and a Fast R-CNN method is applied for object detection using the predicted BBs with a detector based on VGG-16 model. The training process can be regarded as a Four-Step-Alternative-Training [39].

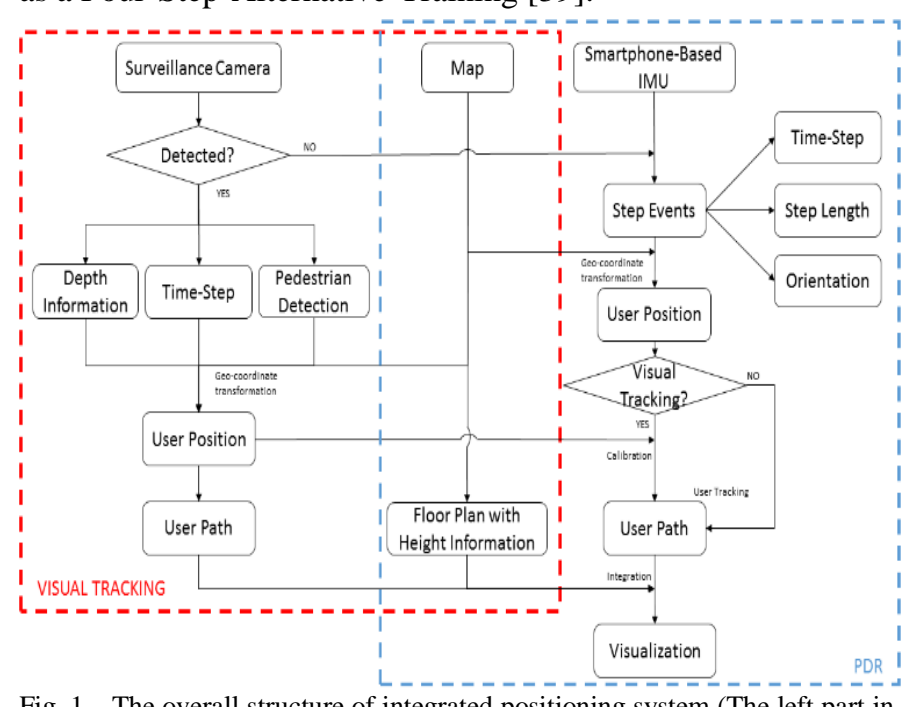

Fig. 1. The overall structure of integrated positioning system (The left part in red box is visual tracking and the right part in blue box is PDR system). 


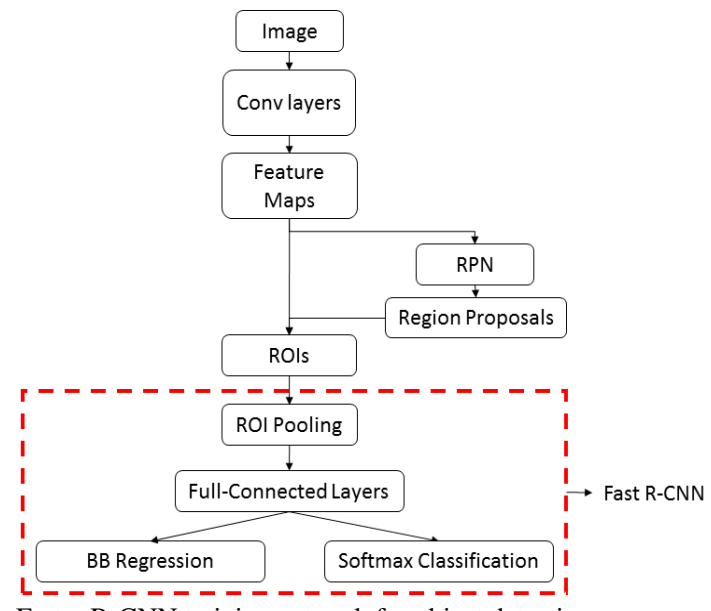

Fig. 2. Faster R-CNN training network for object detection.

In this study, the Faster R-CNN based method uses the pretrained human detector for human based on data from MS COCO and PASCAL VOC 2007 plus 2012. After extracting BBs with frame numbers from video, the central points of their lower boundaries are used as the position of feet, i.e. the lowest member of human body, to produce the user path and the time step is inferred from frame frequency (Fig.3). This study only has one user for pedestrian detection, thus eliminate the effects from mixture of other people, however, it may be a problem in future with multiple users in camera. Meanwhile, due to the relatively initial long distance between the user and the camera, it is likely to miss the user from the detection process in the initial state even in view of the camera.
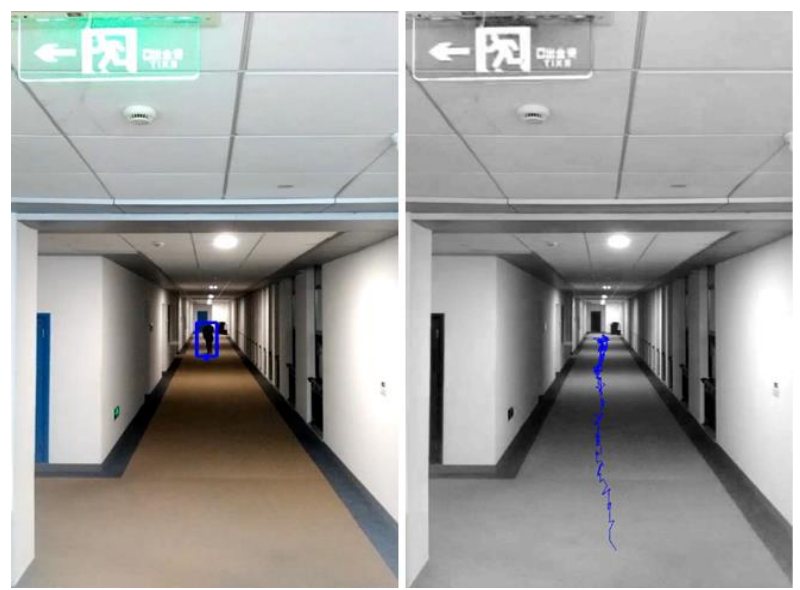

Fig. 3. An example of extracted feet position (left) and the user path (right) from video.

\section{2) Depth Information}

Depth information, i.e. the distance between the user and the camera indicated by $D_{i}$ for $i^{\text {th }}$ frame could be determined by the pixel height $h_{i}$ and real height $H_{p}$ of human as well as focal pixel length $f$. $f$ is determined by camera resolution and field of view (FOV). With the pinhole camera model [40] presented in (1):

$$
\frac{h_{i}}{f}=\frac{H_{p}}{D_{i}}(i=1,2, \ldots, n)
$$

where $D_{i}$ will be proportional to $h_{i}$ and a series of relative user distance to camera will then be calculated.

\section{B. PDR Based User Tracking}

The PDR processing used in this study is based on previous studies utilizing Weinberg's stride length estimation and heading measurement and having transformation of body frame to global frame [41-43].

\section{1) Step Detection}

The step events could be identified by applying a thresholdbased step cycle on accelerations. This follows the principle of stationary inertial sensor during stance and searches for repetitive acceleration data pattern [e.g. 44, 45, 46]. The measured acceleration will be first processed by a low-pass filter [43] with frequency condition [11] depended on accelerometer sampling rate. Then, the norm of accelerations in three axis $\left(a_{x}(t), a_{y}(t)\right.$, and $\left.a_{y}(t)\right)$ is taken from the accelerometer, which can get the vertical changes with respect to the time [47] as denoted in formula (2):

$$
a(t)=\sqrt{\left(a_{x}(t)\right)^{2}+\left(a_{y}(t)\right)^{2}+\left(a_{z}(t)\right)^{2}}-g
$$

where $\mathrm{g}$ is the gravity, which needs to be excluded from vertical acceleration. The acceleration will then be processed by applying threshold to classify acceleration phases, static phases and deceleration phases of gait cycle process. The next step is to apply a zero-crossing method for cyclic property detection [48]. The detected steps after processing acceleration is represented in Fig. 4.

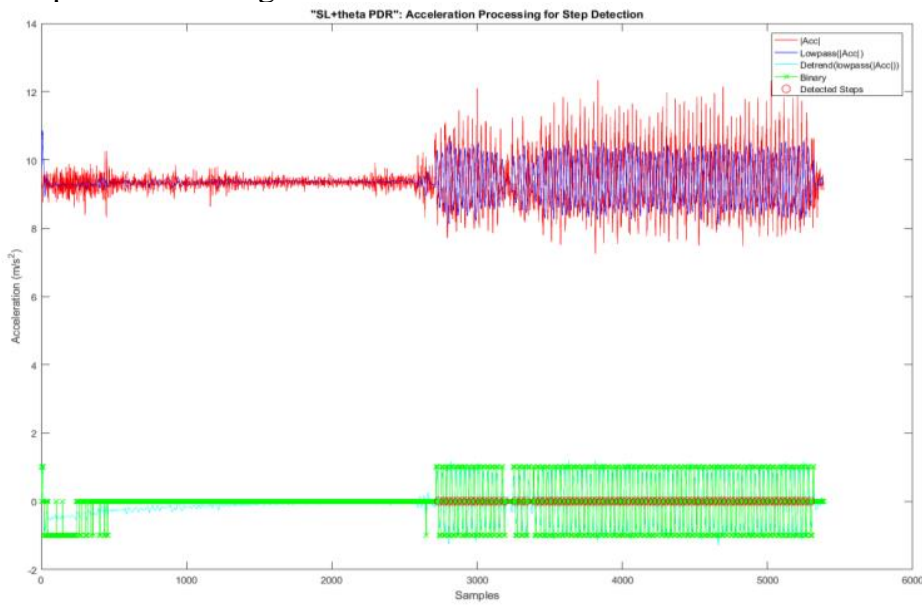

Fig. 4. The detected steps represented in red circles (original acceleration in red line, acceleration filtered by low-pass in indigo line, substraction by gravity in blue line and threshold application in green line).

\section{2) Step Length Estimation}

This study applies a Weinberg's algorithm of bounce-based step length estimation as in (3), and the bounce is determined by maximum $\left(a_{\max }(i)\right)$ and minimum value $\left(a_{\min }(i)\right)$ of each step event:

$$
S L_{i}=\sqrt[4]{a_{\max }(i)-a_{\min }(i)} * k * 2(i=1,2, \ldots, n)
$$

where $S L_{i}$ is the step length of the $i^{t h}$ step and $k$ is an empirical value of penalty for calculation [43].

\section{3) Heading Estimation}

The heading of IMU on hand-held smartphone in global frame can be calculated by modification of angular velocity in body frame with updating rotation matrix $R(t)$ in (4)-(9): 


$$
\begin{aligned}
& R_{x}(t)=\left(\begin{array}{ccc}
1 & 0 & 0 \\
0 & \cos (\phi(t)) & -\sin (\phi(t)) \\
0 & \sin (\phi(t)) & \cos (\phi(t))
\end{array}\right) \\
& R_{y}(t)=\left(\begin{array}{ccc}
\cos (\theta(t)) & 0 & \sin (\theta(t)) \\
0 & 1 & 0 \\
-\sin (\theta(t)) & 0 & \cos (\theta(t))
\end{array}\right) \\
& R_{z}(t)=\left(\begin{array}{ccc}
\cos (\psi(t)) & -\sin (\psi(t)) & 0 \\
\sin (\psi(t)) & \cos (\psi(t)) & 0 \\
0 & 0 & 1
\end{array}\right) \\
& R(t)=R_{z}(t) R_{y}(t) R_{x}(t) \\
& X=\left(\begin{array}{ccc}
0 & -\omega_{z}^{t} \Delta t & \omega_{y}^{t} \\
\omega_{z}^{t} \Delta t & 0 & -\omega_{x}^{t} \Delta t \\
-\omega_{y}^{t} \Delta t & \omega_{x}^{t} \Delta t & 0
\end{array}\right) \\
& R(t+\Delta t)=R(t) * \exp (X)
\end{aligned}
$$

where $R_{x}(t), R_{y}(t)$, and $R_{z}(t)$ represents the rotation matrix from body frame to global frame in roll $\phi(t)$, pitch $\theta(t)$ and yaw $\psi(t)$ directions in body frame respectively, as a function of time $t$. The updating of rotation matrix $R(t)$ is based on angular velocity changes of body frame in three axis as $\omega_{x}^{t}, \omega_{y}^{t}$ and $\omega_{z}^{t}$ at time t. The overall angular rotation within sampling interval $\Delta t$ in these three directions as $X$ will then be applied to the rotation matrix in current state $R(t)$ in order to get the rotation matrix at next state as $R(t+\Delta t)$. The initial states of roll and pitch angle are determined by initial average acceleration changes in same directions and the initial yaw will be zero as the starting point of heading. For simplification, the IMU is assumed to be held stably, and so the heading $\Psi(t)$ is only related to the changes in yaw direction in each step [11, 43] and can be calculated as:

$$
\Psi(t)=\arctan _{2}\left(R_{2,1}(t), R_{1,1}(t)\right)
$$

Using step detection, step length and the heading estimation information, the user position can be calculated as following:

$$
\left[\begin{array}{c}
P_{E_{i}} \\
P_{N_{i}}
\end{array}\right]=\left[\begin{array}{c}
P_{E_{i-1}}+S L_{i} * \sin (\Psi(t)) \\
\left.P_{N_{i-1}}+S L_{i} * \cos (\Psi(t))\right)
\end{array}\right]
$$

Where $P_{E_{i}}$ and $P_{N_{i}}$ represent the position in east and north direction [11, 43]. However, the directions pointed out in PDR system may not be geo-oriented. Thus, an integration of map information is essential for the first step of calibration of PDR based on visual tracking system.

\section{Geographic Information Integration}

In this study, the geographical information will be collected from georeferenced floor plan, which is also regarded as the basemap providing absolute positioning information as well as simplified semantic building information. The reference coordinate system is WGS1984 with prior knowledge of building height of experimental area (9.5 m). WGS1984 makes the integration of indoor system with the outdoor much easier as it is Spatial Reference System (SRS) form GPS transmitted data. Both the paths from visual tracking and PDR must be transferred into same coordinate system for later calibration of PDR.

\section{Calibration of PDR by Visual tracking}

As visual tracking has a relatively higher accuracy than PDR results in LOS area, therefore, the vision-based results can be utilized to calibrate PDR positioning in this area. In this study, a simple method is applied to minimize the computation cost. The method used the time synchronization data from both positioning components. Both PDR and visual data have recorded time steps: the former is deduced from acceleration timestamps and detected step events, and the latter is calculated from frame number and frequency. The PDR results with similar time step in vision-based tracking will then be replaced by corresponding detected feet positions in visual tracking results.

\section{EXPERIMENTS AND RESULTS}

The proposed system is implemented and tested in an experimental site located on the $4^{\text {th }}$ floor of Sir Peter Mansfield Building in University of Nottingham, Ningbo, China. The whole path is $51.84 \mathrm{~m}$, where only a short part $(8.82 \mathrm{~m})$ which is not covered by camera. The rest, which is a long corridor, could be used for testing the proposed system as the integration of PDR $(43.02 \mathrm{~m})$ and visual tracking results. The resolution of installed camera is $960 \times 544$, vertical FOV is $27^{\circ}$, and thus the pixel length for camera is about $1.05 \times 10^{3}$. The smartphone used for PDR is HUAWEI MT7-TL00, with GetSensorData App [43]. The frame frequency is 16 frames per second and the sampling rate of PDR is set to be $100 \mathrm{~Hz}$. The visual tracking path, uncalibrated PDR path and reference path is presented in Fig.5 within floor plan digitized by ArcGIS 10.3.

During the experiment, both PDR and camera recordings start simultaneously with smartphone-based PDR held horizontally, pointing to the heading direction. Over precalibration phase, the Root Mean Square Error (RMSE) of PDR-based positioning is $0.82 \mathrm{~m}$ with significant drift after turning (Fig.5) while over post-calibration, the acquired PDR path from the long corridor part has been improved as shown in Fig.6 with the RMSE of $0.73 \mathrm{~m}$ with a decrease of $10.98 \%$. Comparing calibrated route against reference route, it indicates that this study provides a better solution than using either of individual tracking system as it both takes the advantage of accurate visual tracking in LOS area and the continuity of PDR in out-of-sight area. In addition, the last step event of PDR path has also been calibrated to match with ending point of reference path, which will provide a correct starting point for the following tracking and is helpful to link the partial trajectories of user recorded in the current camera and the next camera. However, the calibrated step positons are not evenly distributed as they used to be, although the calibrated PDR based tracking route has been matched to the reference path. This is basically due to the error caused by pinhole effects from visual tracking during calibration as it will be problematic at the beginning and the ending part of visual positioning. In the initial state, the highly dense positioning will appear with undetectable change of pixel height of human while reaching ending state, the significant change of human's pixel height will lead to sparse positioning based on formula (1). This could lead to an uneven 
distribution of visual tracking based positions, which could be potentially improved by applying a PF-based matching of vision-based tracking and map, as this time only a simple time synchronization is used.
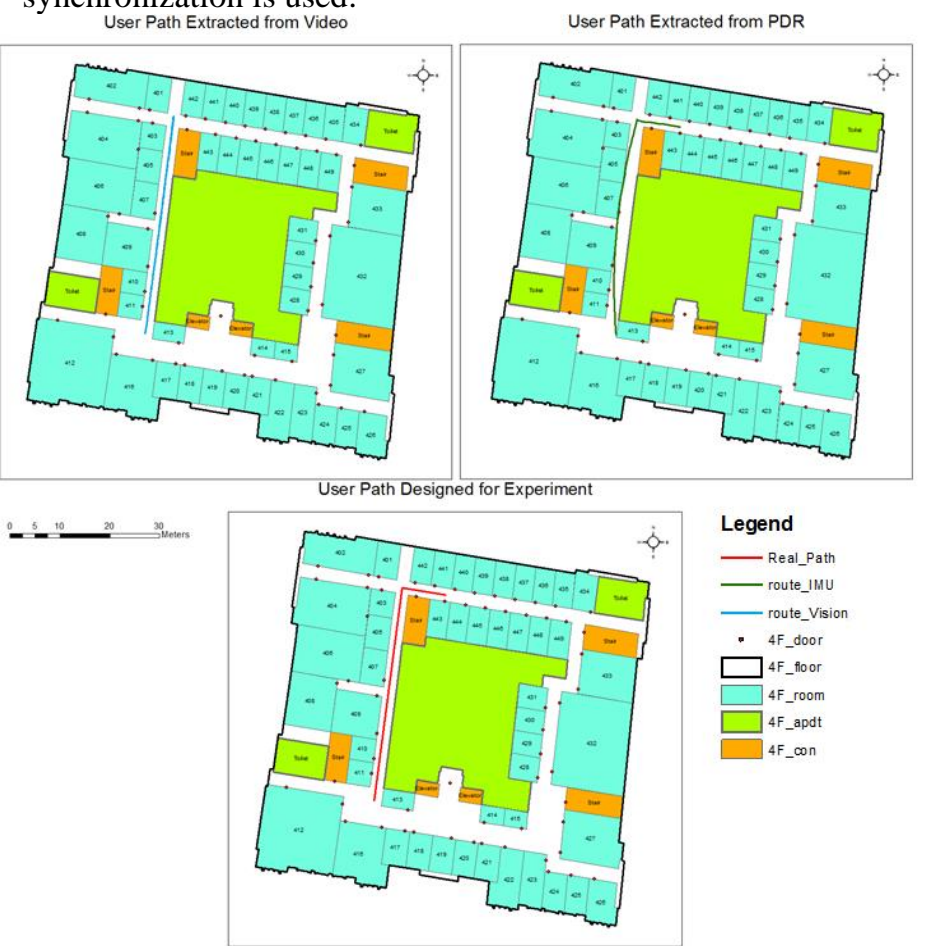

Fig. 5. The georeferenced results of visual tracking path (upper left), PDR path (upper right), and the designed reference path (downside) (with $4 \mathrm{~F}$ _floor, 4F_door, 4F_room, 4F_adpt and 4F_con to represent floor shape, doors, rooms, non-functional places and connections such as staircases and elevators). User Path Extracted from Calibrated PDR

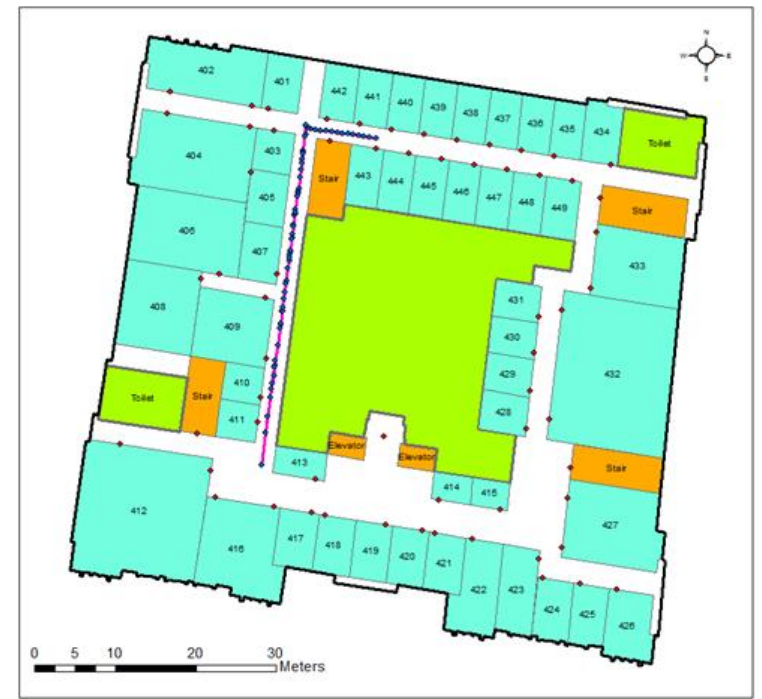

Legend - Imu_cal - route_mu_c - 4F_door $\square_{4 F \text { floo }}$ 4F_floor Fig. 6. Calibrated route and positions of each step event of PDR based positioning.

In future, PDR system will be further utilized for staircasewalking tracking with the support of a barometer for height detection, in order to automatically change the related floor plan while the visual tracking system will utilize a multi-camera system to calibrate the entire PDR-based movements. In addition, this hybrid system will also try to detect multiple users' path at the same time to see whether the system is still functional with the same level of performance, scalability and accuracy. Finally, as the system already applies WGS 1984 coordinate system, an indoor-outdoor scenario can examine the performance and functionality of the system.

\section{CONCLUSION}

This study introduced a prototype of 'low-cost' hybrid indoor positioning system with utilization of static camera and smartphone-based PDR. The tests and implementation of the proposed system provided with more than $10 \%$ improvement of the accuracy, when the floor plan in the form of geo-referenced map was fed into the system. The possibility of implementing this system simply by a mobile device and minimum of device modification could potentially result in a good solution for indoor positioning and navigation applications. The achieved accuracy and practicality can still be improved by using the PF, and applying a multi-camera system and other sensors such as barometers. The proposed system can also be applied for multiuser detection as Faster R-CNN for indoor-outdoor scenarios as it uses geospatial data in the WGS 1984 system.

\section{ACKNOWLEDGMENT}

The author acknowledges the financial support from the International Doctoral Innovation Centre, Ningbo Education Bureau, Ningbo Science and Technology Bureau, and the University of Nottingham. This work was also supported by the UK Engineering and Physical Sciences Re-search Council [grant number EP/L015463/1]

\section{REFERENCES}

[1] Jiang, L., Hoe, L.N., and Loon, L.L.: 'Integrated UWB and GPS location sensing system in hospital environment', in Editor (Ed.)^(Eds.): 'Book Integrated UWB and GPS location sensing system in hospital environment' (IEEE, 2010, edn.), pp. 286-289

[2] Kjærgaard, M.B., Blunck, H., Godsk, T., Toftkjær, T., Christensen, D.L. and Grønbæk, K.: 'Indoor positioning using GPS revisited ', in Editor $(\text { Ed. })^{\wedge}$ (Eds.): 'Book Indoor positioning using GPS revisited ' (Springer, 2010, edn.), pp. 38-56

[3] Vu, A., Ramanandan, A., Chen, A., Farrell, J.A., and Barth, M.: 'Real Time Computer Vision/DGPS-Aided Inertial Navigation System for Lane-Level Vehicle Navigation', IEEE Transactions on Intelligent Transportation Systems, 2012, 13, (2), pp. 899-913

[4] Niu, L.: 'A survey of wireless indoor positioning technology for fire emergency routing', in Editor (Ed.)^(Eds.): 'Book A survey of wireless indoor positioning technology for fire emergency routing' (IOP Publishing, 2014, edn.), pp. 012127

[5] Filonenko, V., Cullen, C., and Carswell, J.: 'Investigating ultrasonic positioning on mobile phones', in Editor (Ed.)^(Eds.): 'Book Investigating ultrasonic positioning on mobile phones' (IEEE, 2010, edn.), pp. 1-8

[6] Maghdid, H.S., Lami, I.A., Ghafoor, K.Z., and Lloret, J.: 'Seamless outdoors-indoors localization solutions on smartphones: implementation and challenges', ACM Computing Surveys (CSUR), 2016, 48, (4), pp. 53

[7] Mourikis, A.I., and Roumeliotis, S.I.: 'A Multi-State Constraint Kalman Filter for Vision-Aided Inertial Navigation', 2007, 22, pp. 3565-3572

[8] Tardif, J.P., George, M., Laverne, M., and Kelly, A.: 'A new approach to vision-aided inertial navigation', in Editor (Ed.) $)^{\wedge}($ Eds.): 'Book A new approach to vision-aided inertial navigation' (2010, edn.), pp. 4161-4168 
[9] Fuchs, C., Aschenbruck, N., Martini, P., and Wieneke, M.: 'Indoor tracking for mission critical scenarios: A survey', Pervasive and Mobile Computing, 2011, 7, (1), pp. 1-15

[10] Harle, R.: 'A Survey of Indoor Inertial Positioning Systems for Pedestrians', IEEE Communications Surveys and Tutorials, 2013, 15, (3), pp. 1281-1293

[11] Racko, J., Brida, P., Perttula, A., Parviainen, J., and Collin, J.: 'Pedestrian Dead Reckoning with Particle Filter for handheld smartphone', in Editor (Ed.) ${ }^{\wedge}$ (Eds.): 'Book Pedestrian Dead Reckoning with Particle Filter for handheld smartphone' (IEEE, 2016, edn.), pp. 1-7

[12] Basiri, A., Lohan, E.S., Moore, T., Winstanley, A., Peltola, P., Hill, C., Amirian, P., and e Silva, P.F.: 'Indoor location based services challenges, requirements and usability of current solutions', Computer Science Review, 2017

[13] Dong-Si, T.C., and Mourikis, A.I.: 'Estimator initialization in visionaided inertial navigation with unknown camera-IMU calibration', in Editor (Ed.) $)^{\wedge}$ Eds.): 'Book Estimator initialization in vision-aided inertial navigation with unknown camera-IMU calibration' (2012, edn.), pp. 1064-1071

[14] Woodman, O.J.: 'An introduction to inertial navigation', in Editor $(\text { Ed. })^{\wedge}($ Eds.): 'Book An introduction to inertial navigation' (University of Cambridge, Computer Laboratory, 2007, edn.), pp.

[15] Rajagopal, S.: 'Personal dead reckoning system with shoe mounted inertial sensors', Master's Degree Project, Stockholm, Sweden, 2008

[16] Abdulrahim, K., Hide, C., Moore, T., and Hill, C.: 'Aiding low cost inertial navigation with building heading for pedestrian navigation', Journal of navigation, 2011, 64, (02), pp. 219-233

[17] Lin, P.C., Lu, J.C., Tsai, C.H., and Ho, C.W.: 'Design and Implementation of a Nine-Axis Inertial Measurement Unit', IEEE/ASME Transactions on Mechatronics, 2012, 17, (4), pp. 657-668

[18] Griesbach, D., Baumbach, D., and Zuev, S.: 'Stereo-vision-aided inertial navigation for unknown indoor and outdoor environments', International Journal of Cultural Policy, 2014, 20, (3), pp. 281-295

[19] Pinchin, J., Hide, C., and Moore, T.: 'The use of high sensitivity gps for initialisation of a foot mounted inertial navigation system', in Editor $(\text { Ed. })^{\wedge}($ Eds.): 'Book The use of high sensitivity gps for initialisation of a foot mounted inertial navigation system' (IEEE, 2012, edn.), pp. 9981007

[20] Panahandeh, G., and Jansson, M.: 'Vision-Aided Inertial Navigation Based on Ground Plane Feature Detection', IEEE/ASME Transactions on Mechatronics, 2014, 19, (4), pp. 1206-1215

[21] Skog, I., Handel, P., Nilsson, J.O., and Rantakokko, J.: 'Zero-Velocity Detection-An Algorithm Evaluation', IEEE transactions on bio-medical engineering, 2010, 57, (11), pp. 2657-2666

[22] Ramanandan, A., Chen, A., and Farrell, J.A.: 'Inertial Navigation Aiding by Stationary Updates', IEEE Transactions on Intelligent Transportation Systems, 2012, 13, (1), pp. 235-248

[23] Mautz, R., and Tilch, S.: 'Survey of optical indoor positioning systems', in Editor $(E d .)^{\wedge}($ Eds.): 'Book Survey of optical indoor positioning systems' (IEEE, 2011, edn.), pp. 1-7

[24] He, C., Kazanzides, P., Sen, H.T., Kim, S., and Liu, Y.: 'An Inertial and Optical Sensor Fusion Approach for Six Degree-of-Freedom Pose Estimation', Sensors, 2015, 15, (7), pp. 16448-16465

[25] Hernandez, J., Tsotsos, K., and Soatto, S.: 'Observability, identifiability and sensitivity of vision-aided inertial navigation', in Editor (Ed.) ${ }^{\wedge}($ Eds.): 'Book Observability, identifiability and sensitivity of vision-aided inertial navigation' (IEEE, 2015, edn.), pp. 2319-2325

[26] Hung, T.N., and Suh, Y.S.: 'Inertial sensor-based two feet motion tracking for gait analysis', Sensors, 2013, 13, (5), pp. 5614-5629

[27] Placer, M., and Kovačič, S.: 'Enhancing indoor inertial pedestrian navigation using a shoe-worn marker', Sensors, 2013, 13, (8), pp. 9836 9859

[28] Parnian, N., and Golnaraghi, F.: 'Integration of a multi-camera vision system and strapdown inertial navigation system (SDINS) with a modified Kalman filter', Sensors, 2010, 10, (6), pp. 5378-5394

[29] Hide, C., Botterill, T., and Andreotti, M.: 'Low cost vision-aided IMU for pedestrian navigation', in Editor $(\text { Ed. })^{\wedge}($ Eds.): 'Book Low cost visionaided IMU for pedestrian navigation' (IEEE, 2010, edn.), pp. 1-7
[30] Hide, C., Botterill, T., and Andreotti, M.: 'Vision-aided IMU for handheld pedestrian navigation', in Editor $(\text { Ed. })^{\wedge}($ Eds. $)$ : ‘Book Vision-aided IMU for handheld pedestrian navigation' (2010, edn.), pp.

[31] Liu, T., Carlberg, M., Chen, G., Chen, J., Kua, J., and Zakhor, A.: 'Indoor localization and visualization using a human-operated backpack system', in Editor $(\text { Ed. })^{\wedge}($ Eds. $)$ : 'Book Indoor localization and visualization using a human-operated backpack system' (IEEE, 2010, edn.), pp. 1-10

[32] Azimi, E., Doswell, J., and Kazanzides, P.: 'Augmented reality goggles with an integrated tracking system for navigation in neurosurgery', in Editor (Ed.) $)^{\wedge}$ Eds.): 'Book Augmented reality goggles with an integrated tracking system for navigation in neurosurgery' (IEEE, 2012, edn.), pp. 123-124

[33] Sadda, P., Azimi, E., Jallo, G., Doswell, J., and Kazanzides, P.: 'Surgical navigation with a head-mounted tracking system and display': 'Studies in Health Technology and Informatics' (IOS Press, 2013)

[34] Konolige, K., Agrawal, M., and Sola, J.: 'Large-scale visual odometry for rough terrain': 'Robotics research' (Springer, 2010), pp. 201-212

[35] Lupton, T., and Sukkarieh, S.: 'Visual-inertial-aided navigation for highdynamic motion in built environments without initial conditions', IEEE Transactions on Robotics, 2012, 28, (1), pp. 61-76

[36] Bleser, G., and Strickery, D.: 'Using the marginalised particle filter for real-time visual-inertial sensor fusion', in Editor $(\mathrm{Ed} .)^{\wedge}(\mathrm{Eds}$.$) : 'Book$ Using the marginalised particle filter for real-time visual-inertial sensor fusion' (IEEE, 2008, edn.), pp. 3-12

[37] Lupton, T., and Sukkarieh, S.: 'Efficient integration of inertial observations into visual SLAM without initialization', in Editor $(\text { Ed. })^{\wedge}$ (Eds.): 'Book Efficient integration of inertial observations into visual SLAM without initialization’ (IEEE, 2009, edn.), pp. 1547-1552

[38] Torres-Solis, J., Falk, T.H., and Chau, T.: 'A review of indoor localization technologies: towards navigational assistance for topographical disorientation' (INTECH Open Access Publisher, 2010. 2010)

[39] Ren, S., He, K., Girshick, R., and Sun, J.: 'Faster R-CNN: Towards realtime object detection with region proposal networks', in Editor $(\text { Ed. })^{\wedge}($ Eds.): 'Book Faster R-CNN: Towards real-time object detection with region proposal networks' (2015, edn.), pp. 91-99

[40] Dollar, P., Wojek, C., Schiele, B., and Perona, P.: 'Pedestrian detection: An evaluation of the state of the art', IEEE transactions on pattern analysis and machine intelligence, 2012, 34, (4), pp. 743-761

[41] Jimenez, A.R., Zampella, F., and Seco, F.: 'Light-matching: A new signal of opportunity for pedestrian indoor navigation', in Editor (Ed.) $)^{\wedge}$ (Eds.): 'Book Light-matching: A new signal of opportunity for pedestrian indoor navigation' (IEEE, 2013, edn.), pp. 1-10

[42] Torres-Sospedra, J., Jiménez, A.R., Knauth, S., Moreira, A., Beer, Y., Fetzer, T., Ta, V.-C., Montoliu, R., Seco, F., and Mendoza-Silva, G.M.: 'The Smartphone-Based Offline Indoor Location Competition at IPIN 2016: Analysis and Future Work', Sensors, 2017, 17, (3), pp. 557

[43] Zampella, F., Jiménez, A.R., and Seco, F.: 'LOPSI repository: GetSensorData application', 2017

[44] Stirling, R., Collin, J., Fyfe, K., and Lachapelle, G.: 'An innovative shoemounted pedestrian navigation system', in Editor (Ed.) $)^{\wedge}($ Eds.): 'Book An innovative shoe-mounted pedestrian navigation system' (2003, edn.), pp. 22-25

[45] Krach, B., and Robertson, P.: 'Cascaded estimation architecture for integration of foot-mounted inertial sensors', in Editor $(\text { Ed. })^{\wedge}($ Eds.): 'Book Cascaded estimation architecture for integration of foot-mounted inertial sensors' (IEEE, 2008, edn.), pp. 112-119

[46] Castaneda, N., and Lamy-Perbal, S.: 'An improved shoe-mounted inertial navigation system', in Editor $(E d .)^{\wedge}(E d s$.$) : 'Book An improved shoe-$ mounted inertial navigation system' (IEEE, 2010, edn.), pp. 1-6

[47] Kang, W., Nam, S., Han, Y., and Lee, S.: 'Improved heading estimation for smartphone-based indoor positioning systems', in Editor (Ed. $)^{\wedge}(E d s$.$) :$ 'Book Improved heading estimation for smartphone-based indoor positioning systems' (IEEE, 2012, edn.), pp. 2449-2453

[48] Goyal, P., Ribeiro, V.J., Saran, H., and Kumar, A.: 'Strap-down pedestrian dead-reckoning system', in Editor $(E d .)^{\wedge}(E d s$.$) : 'Book Strap-$ down pedestrian dead-reckoning system' (IEEE, 2011, edn.), pp. 1-7. 
